\title{
Analysis of the Effect of Bedside Nursing Mode in Treatment of Traumatic Optic Neuropathy with Optic Nerve Decompression
}

\author{
Jie Zhang ${ }^{*}{ }^{\dagger}$, Xiuwen $\mathrm{Li}^{\dagger}$ \\ Email address: \\ zhangjie@jnu.edu.cn (Jie Zhang) \\ ${ }^{*}$ Corresponding author \\ $\dagger$ Jie Zhang and Xiuwen Li are co-first authors.
}

Department of First Class Ward, The First Affiliated Hospital, Jinan University, Guangzhou, China

\section{To cite this article:}

Jie Zhang, Xiuwen Li. Analysis of the Effect of Bedside Nursing Mode in Treatment of Traumatic Optic Neuropathy with Optic Nerve Decompression. Clinical Medicine Research. Vol. 9, No. 2, 2020, pp. 31-34. doi: 10.11648/j.cmr.20200902.11

Received: January 22, 2020; Accepted: February 26, 2020; Published: March 23, 2020

\begin{abstract}
Objective: To explore the effect of bedside work-based nursing mode on psychological stress response and quality of life of patients undergoing nasal endoscopic optic nerve decompression for traumatic optic neuropathy. Methods: Thirty patients with traumatic optic neuropathy were selected from our hospital from March 2016 to October 2019. All patients underwent nasal endoscopic optic nerve decompression and were grouped according to the order of admission. The control group received routine nursing intervention and the observation group had 15 Cases were given bedside nursing intervention based on the control group. The depression (SDS), anxiety (SAS), postoperative quality of life (SF-36) scores, and postoperative clinical effects were compared between the two groups at admission and discharge. Results: The SDS and SAS scores of the observation group at the time of discharge were lower than those of the control group, and the differences were statistically significant $(P<0.05)$. The clinical effect of the observation group was better than the control group, and the total effective rate of the observation group was $93.33 \%(14 / 15)$ which was higher than the $80.00 \%(12 / 15)$ of the control group, the difference was statistically significant $(P<0.05)$. SF-36 scores of physiological function, physical function, emotional function, social function, etc. in the observation group were higher than those in the control group, and the difference was statistically significant. $(P<0.05)$. Conclusion: Treatment of traumatic optic neuropathy with bedside work-based nursing mode by nasal endoscopic optic nerve decompression has significant effects, which can improve their psychological stress response and quality of life with high safety.
\end{abstract}

Keywords: Bedside Nursing Mode, Traumatic Optic Neuropathy, Optic Nerve Decompression Under Nasal Endoscope, Psychological Stress Response, Quality of Life

\section{Introduction}

Traumatic optic neuropathy (TON) refers to the loss of vision without external or initial fundus endoscope or optic nerve damage after trauma [1]. It is a common complication of closed craniocerebral trauma, and the main pathological change is optic nerve tube fracture. Deformation, which directly causes primary damage or trauma to the optic nerve, such as contusion, laceration, bleeding, etc., causing partial or full blood supply to the optic nerve to secondary cause edema and ischemia of the optic nerve, microcirculation disorders cause optic nerve degeneration, retinal ganglion cell necrosis and Apoptosis, causing vision loss or permanent blindness. Optic nerve tube decompression is one of the main treatment methods at present, its purpose is to prevent or reduce secondary damage to the optic nerve, and provide necessary conditions for the recovery of primary damage [2-4]. Transnasal endoscopic optic nerve tube decompression is receiving more and more attention in the clinic because of less damage, accurate curative effect, and no facial scar. However, due to the serious lack of understanding of the disease and decompression of the optic nerve under nasal endoscope, patients are prone to produce psychological stress reactions such as depression, tension, anxiety, and anxiety, which will adversely affect the effect of surgical treatment. 
Surgical care interventions are critical. This article explores the effect of bedside work-based nursing mode on psychological stress and quality of life during hospitalization for patients undergoing nasal endoscopic optic nerve decompression. The report is as follows.

\section{Materials and Methods}

\subsection{General Information}

Thirty patients with traumatic optic neuropathy were selected from our hospital from March 2016 to October 2019. All patients underwent nasal endoscopic optic nerve decompression surgery and were grouped according to the order of admission. The control group consisted of 15 cases, 4 women and 11 men. Aged 15 to $71(49.32 \pm 13.25)$ years old; causes of illness: 4 cases of mechanical injuries, 6 cases of blow injuries, 3 cases of traffic accident injuries, 2 cases of other causes. There were 15 patients in the study group, 5 females and 10 males; aged 14 to $73(49.65 \pm 14.31)$ years; causes of disease: 5 cases of mechanical injuries, 6 cases of blow injuries, 3 cases of traffic accident injuries, and 1 case of other causes. There was no significant difference in clinical data between the two groups $(P>0.05)$, which was comparable. The research obtained the consent of the clinical department and the support of the Medical Ethics Association. The case selection was based on the voluntary principle, and the patients and their families signed informed consent. The study subjects agreed to participate in the psychological state assessment, and recorded the contact information of the patients and important relatives, such as telephone and WeChat for follow-up.

\subsection{Method}

General anesthesia was intubated, and a nasal mucosal surface anesthesia was performed under Wolf sinus endoscope using 15\% 1\% tetracaine plus 1: 1000 epinephrine. The entire ethmoid sinus was opened according to the Messerklinger technique, and the anterior wall of the sphenoid sinus was opened and enlarged. Remove blood clots and bone fragments, recognize anatomical signs, carefully check the sphenoid sieve top, paper template, and sphenoid sinus outer wall for bone destruction, look for optic nerve tube protrusions and internal carotid artery bulges, electric drill to grind the inner wall of optic nerve tube The surgical cavity was intermittently flushed with normal saline to prevent the electric drill from damaging the optic nerve. Use a stripper to carefully remove the back of the paper template and the inner wall of the optic nerve tube for about $1 / 3$ to $1 / 2$ circumference. The optic nerve should not be used as a support for the stripper when removing bone. Pay attention to clean up the fracture fragments and hematoma around the optic cavity and the optic nerve. When dissecting the optic nerve sheath, avoid the ophthalmic artery below the optic nerve and cut the common key ring at the same time. Gentamicin and dexamethasone gelatin sponges were placed on the inside of the optic nerve of the open tube segment. The surgical cavity was filled with an expansion sponge. After 48 hours, the expansion sponge was removed and the surgical cavity was cleaned after 1 week. The control group was given routine nursing interventions, including evaluation of the condition, preoperative preparation, routine psychological enlightenment, and postoperative basic nursing. The study group provided bedside nursing intervention based on the control group, which refers to adjusting the nurse's shift mode according to the actual needs of the patient's condition, assigning each inpatient to the responsible nurse, and moving the nurse station. Into the ward. Nursing staff provide full-care services at the patient's bedside; using a mobile multifunctional nursing car, all the things that must be or may be used in the treatment and nursing process should be prepared in the car, and the patient's vital signs, consciousness. The pupil changes are observed in real time. Specifically include: (1) Strengthening psychological care and health education before surgery: The nurses will patiently and carefully explain the causes of traumatic optic neuropathy, treatment options and related diseases through friendly and friendly communication with patients, focusing on the optic nerve under nasal endoscope Decompression methods, procedures, precautions, and safety. At the same time, comprehensively and accurately evaluate the patient's psychological state before surgery, analyze the causes of psychological stress reactions, and provide targeted psychological intervention according to specific reasons, thereby effectively improving patients' depression and tension., Anxiety, anxiety and other psychological stress reactions, so that they accept the operation in the best psychological state. (2) Strengthen preoperative preparations: Nursing staff will lead patients to complete the examination of various physiological indicators before surgery, and prepare relevant equipment and instruments for nasal endoscopic optic nerve decompression surgery. (3) Strengthen nursing of postoperative complications: a, Cerebrospinal fluid rhinorrhea care: Improper ethmoid sinus opening, skull base fracture and dural tear can cause cerebrospinal fluid rhinorrhea, so the nursing staff should closely observe the amount of nasal exudate and Nature, once it is found that the clear liquid enters the pharyngeal cavity from the posterior nostril, it can be judged as a cerebrospinal fluid nasal leak; $b$, intraorbital infection care: because nasal endoscopic optic nerve decompression requires opening the ethmoid sinus and opening the bone wall of the optic nerve tube, it will Increasing the risk of concurrent intraorbital infections. Nursing staff should closely observe patients for symptoms such as decreased vision, headache, fever, and vomiting. If any of the above symptoms are found, they should be reported to the doctor in charge for proper treatment; c, Nasal bleeding care: Nursing staff should be clear Instruct patients not to rub their noses hard and eat stiff and irritating food, and to lower their heads as little as possible within two days after surgery to prevent nasal bleeding. If the patient is found to continuously emit bright red liquid in the nose or frequent swallowing movements, they should immediately report to the supervisor. The doctor will handle it properly; $\mathrm{d}$, Asphyxia care: the nurse will help the patient to keep the head and the side rest. Remove 
oral and nasal secretions, so as to prevent apnea, and close observation of oxygen saturation.

\subsection{Observation Indicators}

(1) Comparison of depression and anxiety stress scores on admission and discharge between the two groups, using the self-rating anxiety scale (SAS) and self-rating depression scale (self-prepared by Zung [5]) -rating depression scale (SDS) to assess patients' anxiety and depression. Both Chinese version of SAS and SDS have been proved to have good reliability and validity. SAS includes 20 items, with a total score of 50 to 59 points, 60 to 69 points, and higher than 69 points are considered mild, moderate, and severe anxiety respectively; SDS includes 20 items, with a total score of 53 to 62 points, 63 to 72 points or higher than 72 points is considered mild, moderate, or severe depression, respectively. (2) Comparing the clinical effects of the two groups, the ineffectiveness is an increase in visual acuity less than level 1; the remission is an increase in visual acuity 1-2 levels; the cure is normal vision. (3) Compare the incidence of postoperative complications (including cerebrospinal fluid rhinorrhea, intraorbital infection, nasal bleeding, and asphyxia) between the two groups. (4) Before and after the intervention, reference was made to the four dimensions of physiological function, physical function, emotional function, and social function in the Quality of Life (SF-36) Concise Scale [6, 7] to evaluate the quality of life in the two groups.

\subsection{Statistical Processing}

SPSS23.0 software was used for statistical comparison of data processing. Count data was expressed as a percentage $(\%)$. $\chi^{2}$ test was used. Rank data was used for rank sum test. Measurement data was expressed as mean \pm standard deviation $(\mathrm{x} \pm \mathrm{s}) . P<0.05$ was considered statistically significant.

\section{Results}

\subsection{Comparison and Comparison of SAS and SDS Scores Between the Two Groups}

There was no significant difference in SAS and SDS scores between the two groups at admission $(P>0.05)$. The SAS scores and SDS scores of the observation group at discharge were significantly lower than those of the control group $(P<$ 0.05). See Table 1.

Table 1. SAS and SDS score results and comparison between the two groups $(x \pm s)$.

\begin{tabular}{|c|c|c|c|c|c|}
\hline \multirow{2}{*}{ Group } & \multirow{2}{*}{ Cases } & \multicolumn{2}{|l|}{ SAS Score } & \multicolumn{2}{|l|}{ SDS Score } \\
\hline & & Admission & Discharge & Admission & Discharge \\
\hline Observation & 15 & $63.68 \pm 7.92$ & $40.12 \pm 5.32$ & $61.56 \pm 7.31$ & $39.42 \pm 5.21$ \\
\hline Control & 15 & $61.53 \pm 6.81$ & $50.12 \pm 5.13$ & $60.23 \pm 6.12$ & $49.13 \pm 6.52$ \\
\hline $\mathrm{p}$ value & & $>0.05$ & $<0.05$ & $>0.05$ & $<0.05$ \\
\hline
\end{tabular}

\subsection{Comparison of Clinical Efficacy Between the Two Groups}

The total effective rate of the study group was $93.33 \%(14 / 15)$, which was higher than the control group of $80.00 \%$ (12/15). The difference was statistically significant $(P<0.05)$. See Table 2 .

Table 2. Comparison of clinical efficacy between the two groups [n (\%)].

\begin{tabular}{|c|c|c|c|c|c|}
\hline Group & $\mathbf{n}$ & Invalid & Relieved & Healing & Total efficiency \\
\hline Observation & 15 & 1 & 6 & 8 & $14(93.33)$ \\
\hline Control & 15 & 3 & 7 & 5 & $12(80.00)$ \\
\hline $\mathrm{Z} / \chi^{2}$ value & & 1.574 & & 4.365 & \\
\hline $\mathrm{p}$ value & & $<0.05$ & & $<0.05$ & \\
\hline
\end{tabular}

\subsection{Results and Comparison of Postoperative Quality of Life in the Two Groups}

The postoperative quality of life scores in the observation group were significantly higher than those in the control group $(P<$ 0.05). See Table 3.

Table 3. Evaluation results of postoperative quality of life between two groups $(x \pm s)$.

\begin{tabular}{llllll}
\hline Group & Cases & Physiology & Body & Emotion & Society \\
\hline Observation & 15 & $78.23 \pm 15.43$ & $79.45 \pm 16.34$ & $81.76 \pm 19.12$ & $78.76 \pm 4.12$ \\
Control & 15 & $62.76 \pm 10.24$ & $56.31 \pm 15.32$ & $71.44 \pm 12.54$ & $66.64 \pm 4.97$ \\
p value & & $<0.05$ & $<0.05$ & $<0.05$ & $69.11 \pm 3.21$ \\
\hline
\end{tabular}

\section{Discussion}

Traumatic optic neuropathy can cause optic nerve damage, vasospasm, and poor blood flow, which in turn cause optic nerve ischemia and even necrosis, adversely affects visual function [8, 9]. At present, nasal endoscopic optic nerve decompression is often used in clinical treatment, but due to sudden onset and serious damage, coupled with insufficient preparation for visual function impairment and discomfort caused by surgery, it is easy to produce negative emotions and lead to treatment compliance decline. Moreover, the visual 
function of the patient decreased rapidly in a short period of time, resulting in psychological stress reactions such as depression, tension, anxiety, and anxiety. Currently, nursing work is particularly important. Bedside work-based nursing is one of the commonly used clinical nursing modes. It is guided by holistic nursing theory, takes nursing procedures as the core, and uses tube bed responsibility system + group responsibility system to effectively Integrate the content of nursing work, place commonly used nursing supplies in a multifunctional nursing car as a mobile nurse station, move the nurse station to the ward, allow the nurse to perform all nursing work based on the ward, reduce the time for the nurse to leave the clinical environment, and enable the nurse. Most of the nursing time is provided with direct care services for patients to improve the quality of care which can significantly improve the quality of care and patient care satisfaction. We facilitate language communication between nurses and patients through bedside work. The bedside work system is implemented. The working place of the nurse is in the ward, which is closer to the patient in space than working in the nurse station or treatment room. It is easier to form a close patient-patient relationship with the patient, as in the same office [10]. It is easier for the staff or neighborhood to form close interpersonal relationships, and the nurses spend most of their time in the ward. Naturally, activities such as talking, greeting, comforting, asking, notifying, and instructing with patients and their families are more frequent. It is also conducive to strengthening preoperative health education and psychological nursing intervention, effectively improving their psychological stress response, enabling them to undergo surgery in the best psychological state, improving their adaptive capacity, and thus promoting their rehabilitation process. The bedside work system can be used to enhance the nursing of complications after surgery. Patients are always advised not to rub the nose and eat hard, irritating food, and to lower their heads as little as possible within 2 days after surgery, which can effectively prevent nasal bleeding. The patient's condition changes and can be treated in time. Treatments such as oxygen inhalation, removal of oral cavity and nasal secretions can effectively prevent suffocation. Since the ethmoid sinus needs to be opened and the bone wall of the optic nerve tube is opened during surgery, the orbit will increase the risk of internal infections can be closely monitored by bedside nursing staff for symptoms such as headache, fever, and vomiting. If found, they can immediately report to the attending doctor for proper treatment, which can significantly improve the patient's prognosis by effectively preventing postoperative complications. This study showed that the SDS and SAS scores of the observation group were lower than those of the control group after intervention, the clinical effect was better than that of the control group, the total effective rate was higher than that of the control group, and the postoperative quality of life was higher than that of the control group.
Surgical treatment of patients with traumatic optic neuropathy at bedside work mode has a significant effect and can significantly improve their psychological stress response.

In short, the treatment of bedside work-based nursing mode for patients with traumatic optic neuropathy by nasal endoscopic optic nerve decompression has a significant effect, which can significantly improve their psychological stress response and quality of life, promote patient recovery, and have higher safety. Higher clinical application significance. However, the sample size of this study is small, and it is necessary to expand the sample for further research in the future to verify the conclusion and provide effective nursing methods for improving the clinical efficacy of postoperative nasal endoscopic optic nerve decompression in patients with traumatic optic neuropathy.

\section{Acknowledgements}

The authors declare no conflict of interest.

\section{References}

[1] Welkoborsky, H. J., et al., [Traumatic optic nerve neuropathy. Longterm results following microsurgical optic nerve decompression]. HNO, 2011. 59 (10): p. 997-1004.

[2] Samardzic, K., et al., Traumatic optic neuropathy - to treat or to observe? 2012. 20 (2): p. 131-132.

[3] Guy, W. M., et al., Traumatic Optic Neuropathy and Second Optic Nerve Injuries. Jama Ophthalmol. 132 (5): p. 567.

[4] Peng, A., et al., Endoscopic optic nerve decompression for traumatic optic neuropathy in children. 75 (8): p. 0-998.

[5] Shu, L., A self-rating depression scale. Chin Ment Health J, 1999. 13: p. 194-196.

[6] Tumor site and disease stage as predictors of quality of life in head and neck cancer: a prospective study on patients treated with surgery or combined therapy with surgery and radiotherapy or radiochemotherapy. European Archives of Oto-Rhino-Laryngology. 273 (1): p. 215-224.

[7] Maringwa, J. T., et al., Minimal important differences for interpreting health-related quality of life scores from the EORTC QLQ-C30 in lung cancer patients participating in randomized controlled trials. 19 (11): p. 1753-1760.

[8] Yu-Wai-Man and Patrick, Traumatic optic neuropathy Clinical features and management issues. Taiwan Journal of Ophthalmology. 5 (1): p. 3-8.

[9] Tumor-associated mutations in $<\mathrm{i}>\mathrm{O}</ \mathrm{i}>6$-methylguanine DNA-methyltransferase (MGMT) reduce DNA repair functionality. Molecular Carcinogenesis, 2014. 53 (3): p. 201-210.

[10] Jones and S. F, Communication Skills Training for Health Professionals: An instructorl"s handbook. 76 (8): p. 493. 